\title{
INTEGRATED CAMERA-BASED NAVIGATION
}

\author{
Brita Helene Hafskjold ${ }^{1,2}$, Bjørn Jalving ${ }^{1}$, Per Espen Hagen $^{1}$ and Kenneth Gade ${ }^{1}$ \\ ${ }^{1}$ Norwegian Defence Research Establishment (FFI) \\ P.O. Box 25 \\ N-2027 Kjeller \\ Norway \\ ${ }^{2}$ Center for Technology at Kjeller (UniK) \\ P.O. Box 70 \\ N-2027 Kjeller \\ Norway
}

britah@unik.no, Bjorn.Jalving@ffi.no,Per-Espen.Hagen@ffi.no, Kenneth.Gade@ffi.no

\begin{abstract}
This paper presents an integrated INS (Inertial Navigation System) and camera-based navigation system. The camera-based navigation system provides position measurement aiding to the INS. This is an alternative to the conventional GPS (Global Positioning System) aided INS. The system is intended for UAVs (Unmanned Aerial Vehicles) and long range missiles.

The basic principles of camera-based navigation are presented. The Kalman filter based integration of INS and camera-based navigation is discussed. Total system simulation results are shown together with INS simulations for comparison. Finally, a brief overview of factors that improve the navigation accuracy is presented.
\end{abstract}

\section{Introduction}

GPS is vulnerable to jamming and other disturbances. Camera-based navigation cannot that easily be disturbed, and an INS is impossible to jam. By combining camera-based navigation and INS in an integrated navigation system, we obtain a system that unites:

- the precise measurement of high frequency motion that the INS delivers, and

- the low position drift rate that the camera-based navigation offers.

The system contains three types of sensors:

- A camera (strapdown), capturing the terrain. The raw image data are fed into an imageprocessing algorithm, the output of which is a set of recognisable points in the image. The camera-based navigation is described in more detail in Chapter 3.

- An IMU (Inertial Measurement Unit) containing three gyroscopes and three accelerometers.

- An altimeter (e.g. an air pressure transmitter). 
An existing digital elevation map is also used.

This work is based on the camera-based navigation found in Hagen [1] and the INS work in Gade [2]. The contribution of this work is the integration of these two systems. Two textbooks on INS which can be recommended are Britting [3] and Titterton \& Weston [4].

\section{Mathematical notation}

The mathematical notation used in this paper is:

$p$ image token

$l$ landmark

$\hat{x}$ estimate of $x$

$\tilde{x}$ measurement of $x$

$x_{k}$ value of $x$ at time $k$

$\boldsymbol{x}^{A}$ vector $x$ decomposed in co-ordinate system $A$
$I$ inertial co-ordinate system

$L$ local co-ordinate system

$C$ camera co-ordinate system

$B$ body co-ordinate system

$E$ earth co-ordinate system

$P$ picture co-ordinate system

The co-ordinate systems used in this paper are depicted in Figures 1 and 2:

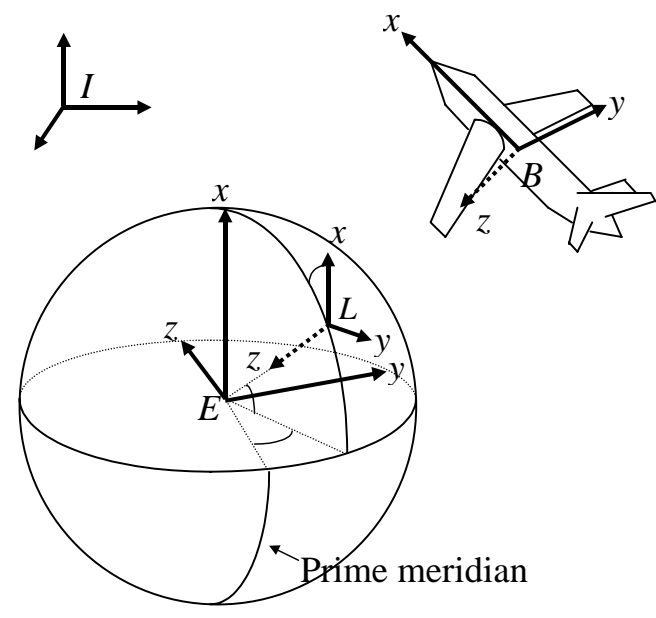

Figure 1 Co-ordinate systems B, I, E and L.
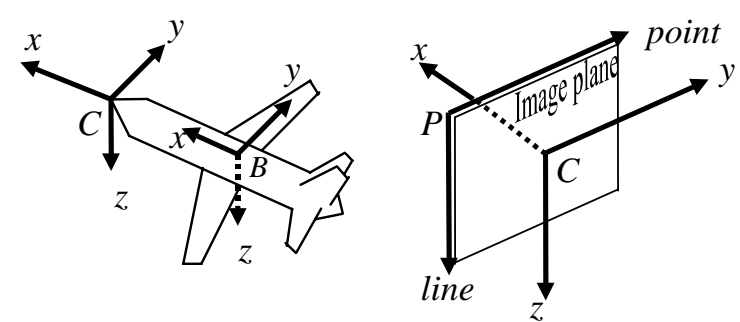

Figure 2 Co-ordinate systems $B, C$ and $P$.

\section{Principles of camera-based navigation}

Camera-based navigation has traditionally been used to navigate across a pre-mapped terrain, which means that a flight must be performed over the specific route to pre-collect either easyto-recognise structures in the picture or whole pictures. During the subsequent flights, the camera pictures are compared to these pre-saved pictures, and the position and attitude of the plane can be calculated.

Our system does not require pre-mapping. The easy-to-recognise objects must be chosen, and their ground position determined, during flight. This is done by the image processing routine, which chooses an image token as a point in the image that will be easy to recognise in the next image in the sequence. These image tokens' movements in the image give us information 
about the topography of the depicted landscape (and the vehicle movement). The use of this information, which is dependent on the position of the camera relative the terrain, together with an elevation map, gives us a method that is quite different from the traditional "optical flow" algorithm. The image processing routine is fully explained in Hagen [1]. A landmark is a point on the terrain surface that corresponds to an image token. In our system, INS position and attitude are used to calculate the landmark position, as illustrated in Figure 3.

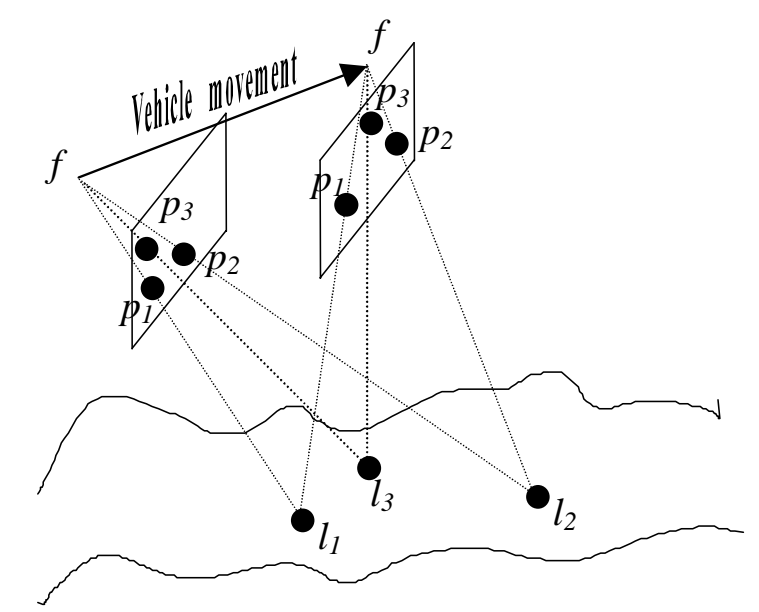

Figure 3 Principles of the camera-based navigation used in this paper $f=$ focal point of the camera, $p_{i}=$ image token no. $i, l_{i}=$ landmark no. $i$

The image tokens will remain in the image as long as the corresponding landmarks are in the line-of-sight of the camera, i.e. within the camera aperture angle. When an image token disappears from the image, a new landmark must be chosen. Image tokens can also be lost because of changes in perspective, where a landmark can be hidden behind a hill, scene changes (such as cars driving down a road), or due to mist or other circumstances that renders the image tokens unrecognisable in the next image. Because we want to keep a constant number of landmarks, a new landmark is chosen each time an image token is lost. This is conducted by the image processing routine, as described above.

\section{Integration of INS and camera-based navigation}

The integrated system works as follows: At each timestep, the INS computes an estimate of the vehicle position and attitude. The integration with the camera-based navigation is carried out by using these estimates together with the position of the landmarks to calculate predicted positions of the image tokens. The difference between these predicted positions and the measured positions of the image tokens is used by an error-state Kalman filter to estimate the INS errors, as illustrated in Figures 4 and 5. 


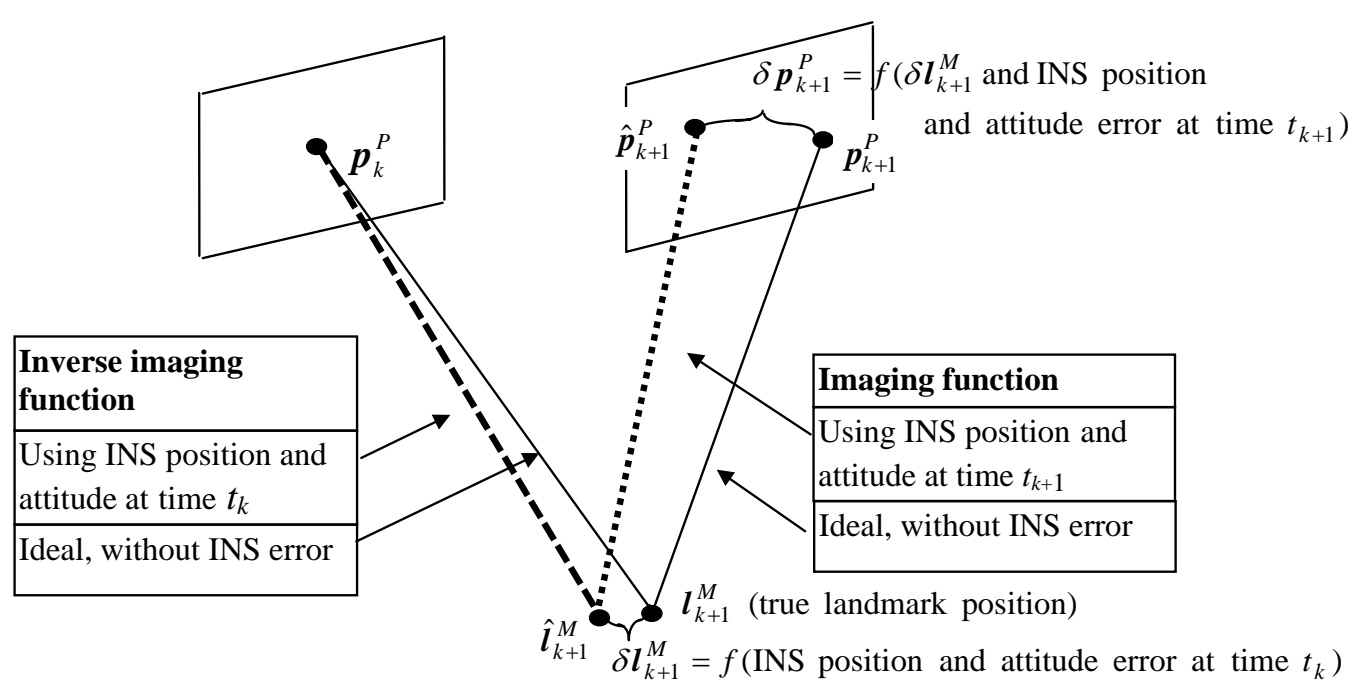

Figure 4 The difference between the calculated $\left(\hat{\boldsymbol{p}}_{k+1}^{P}\right)$ and the true $\left(\boldsymbol{p}_{k+1}^{P}\right)$ image token position is a function of the INS error

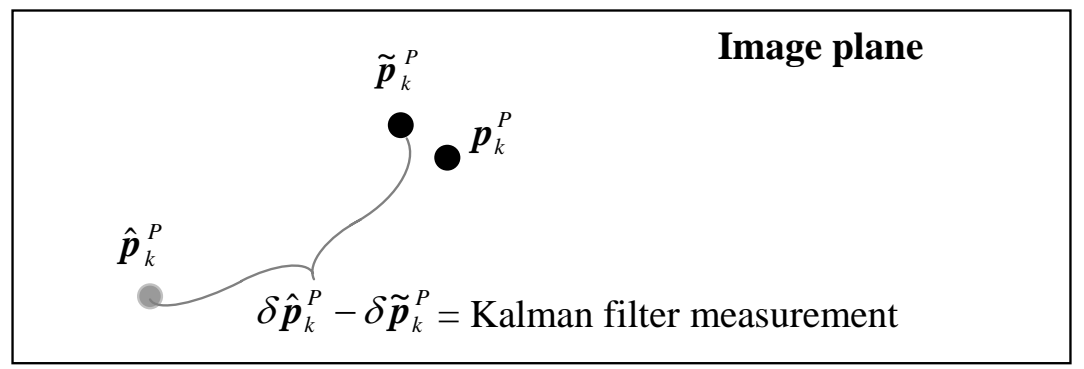

Figure 5 The difference between the calculated ( $\left.\hat{\boldsymbol{p}}_{k}^{P}\right)$ and the measured ( $\left.\tilde{\boldsymbol{p}}_{k}^{P}\right)$ image token position is used as the Kalman filter measurement.

Figure 6 shows the total system structure. The camera-based navigation uses the measurements from the INS and the altimeter to calculate the image token positions in the next image, as illustrated in Figure 4.

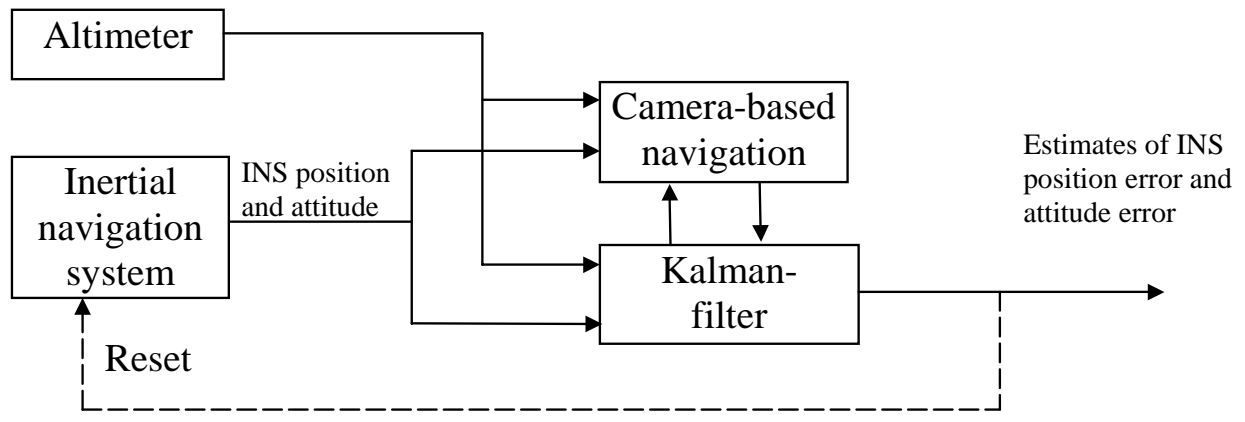

Figure 6 Total system structure 
Figure 7 shows a more detailed total structure picture. The rightmost box represents the image processing routine (explained in Hagen [1]), which measures the position of the image tokens in the next image. The Kalman filter uses the INS attitude and position estimates in its system matrix. The Kalman filter's estimate of the INS attitude and position error is used to reset the INS for each new image in the video stream.

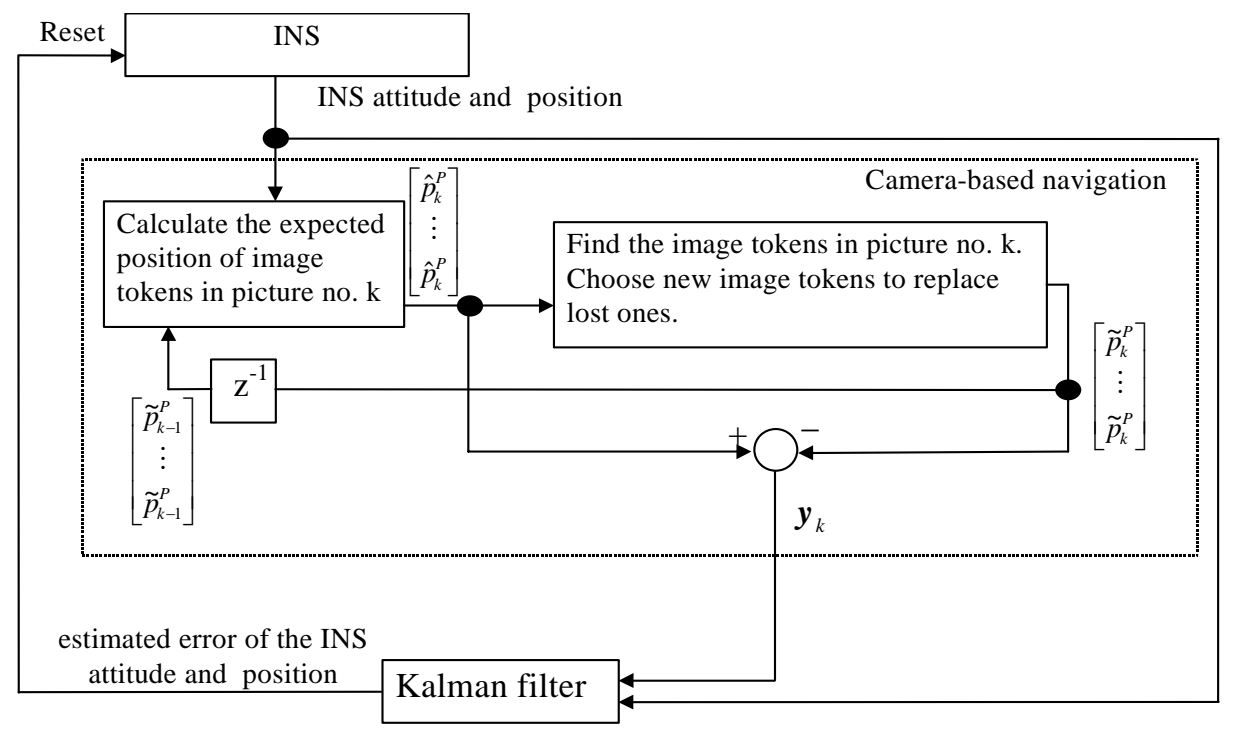

Figure 7 Main algorithm of the camera-based navigation

\section{Simulation results}

The total system has been simulated with the IMU quality, terrain and trajectory given in Chapter 5.1. Chapter 5.2 shows the INS drift (corresponding to the IMU quality in Table 1) and two simulations are shown and briefly discussed in Chapter 5.3. A description of system behaviour is given in Chapter 5.4.

\subsection{Simulation assumptions}

We have made no assumptions as to the type of aerial vehicle used in our simulations. Because our system use the navigation equations for the IMU/INS, the only vehicle parameters used are speed, position and attitude.

\subsubsection{IMU quality}

Table 1 shows the accuracy of the $>10 \mathrm{nmi} / \mathrm{h}$ IMU used in the simulations. 


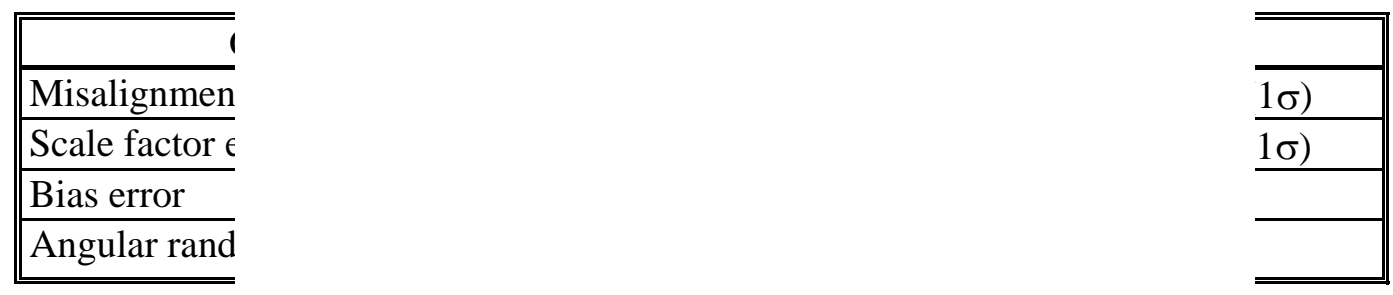

Table 1 IMU

5.1.2 Terrain :

Figure 8 show

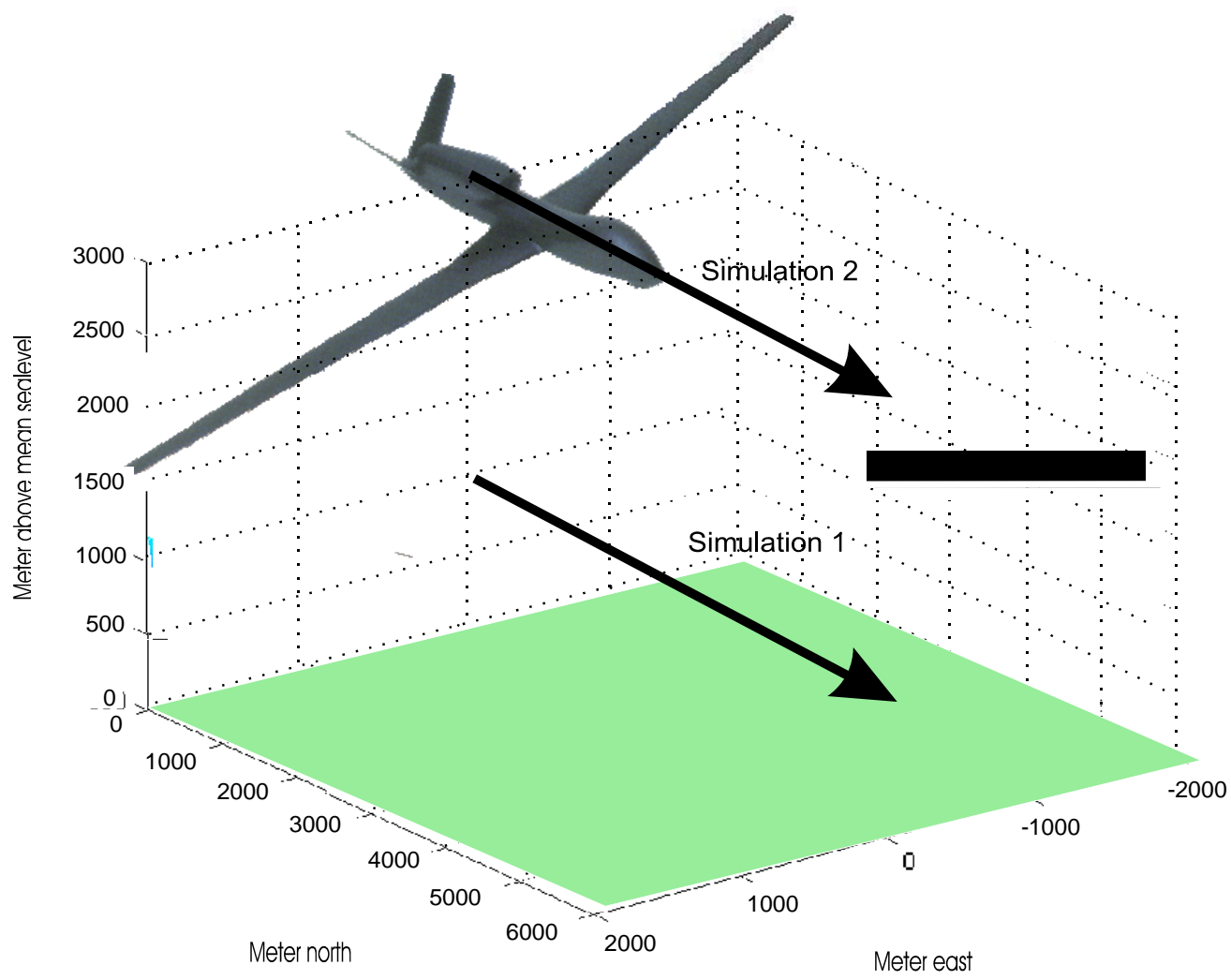

Figure 8 Simulation trajectories 
The UAV starts out at the intersection between the prime meridian and equator, flying straight north with a speed of $50 \mathrm{~m} / \mathrm{s}$. The vehicle height is 1000 meters above mean sea level in simulation 1 and 3000 meters above mean sea level in simulation 2.

\subsection{INS drift}

Figure 9 shows a simulation of the free inertial performance of the INS. The simulation is performed with the IMU quality and trajectory as described in Chapter 5.1.
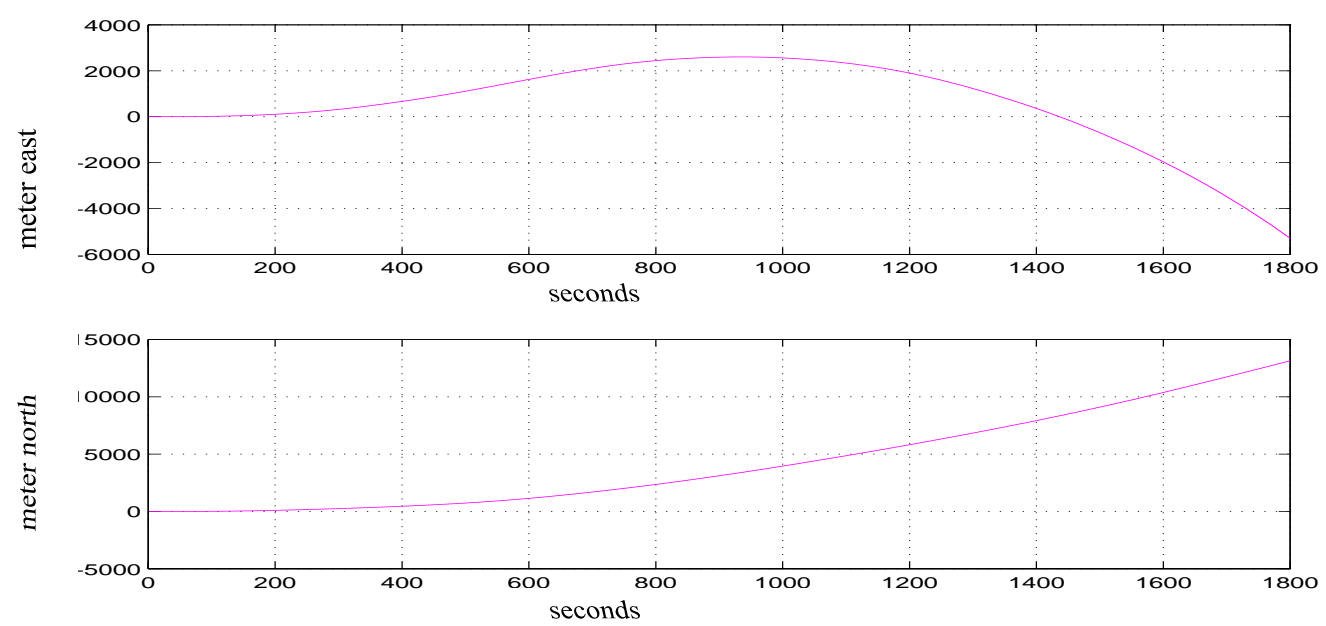

\section{Figure 9 INS positioning error}

\subsection{Total system accuracy}

In this chapter, the simulations use the same IMU quality and trajectory as in Chapter 5.2, but the INS is now aided by the camera-based navigation.

The total system accuracy depends on numerous parameters. In order to illustrate the influence of such parameters on the total system behaviour, only one parameter, namely the vehicle height above the terrain, is changed from simulation 1 to simulation 2 . The rest of the main simulation parameters are:

Picture frequency: $25 \mathrm{~Hz}$

Picture size: $500 \times 500$ pixels

Camera aperture angle: $136^{\circ}$

Camera mounting: Downward, such that the $x$ axis of co-ordinate system $C$ and the $z$ axis of coordinate system $B$ are parallel
Measurement noise: 0.8 pixels

Altimeter error std. deviation: $1 \mathrm{~m}$

Number of landmarks: 12

The influence on the total system behaviour of altering these parameters is briefly discussed in Chapter 5.4.

Figure 10 shows simulation 1 , where the vehicle height is 1000 meters above mean sea level. 

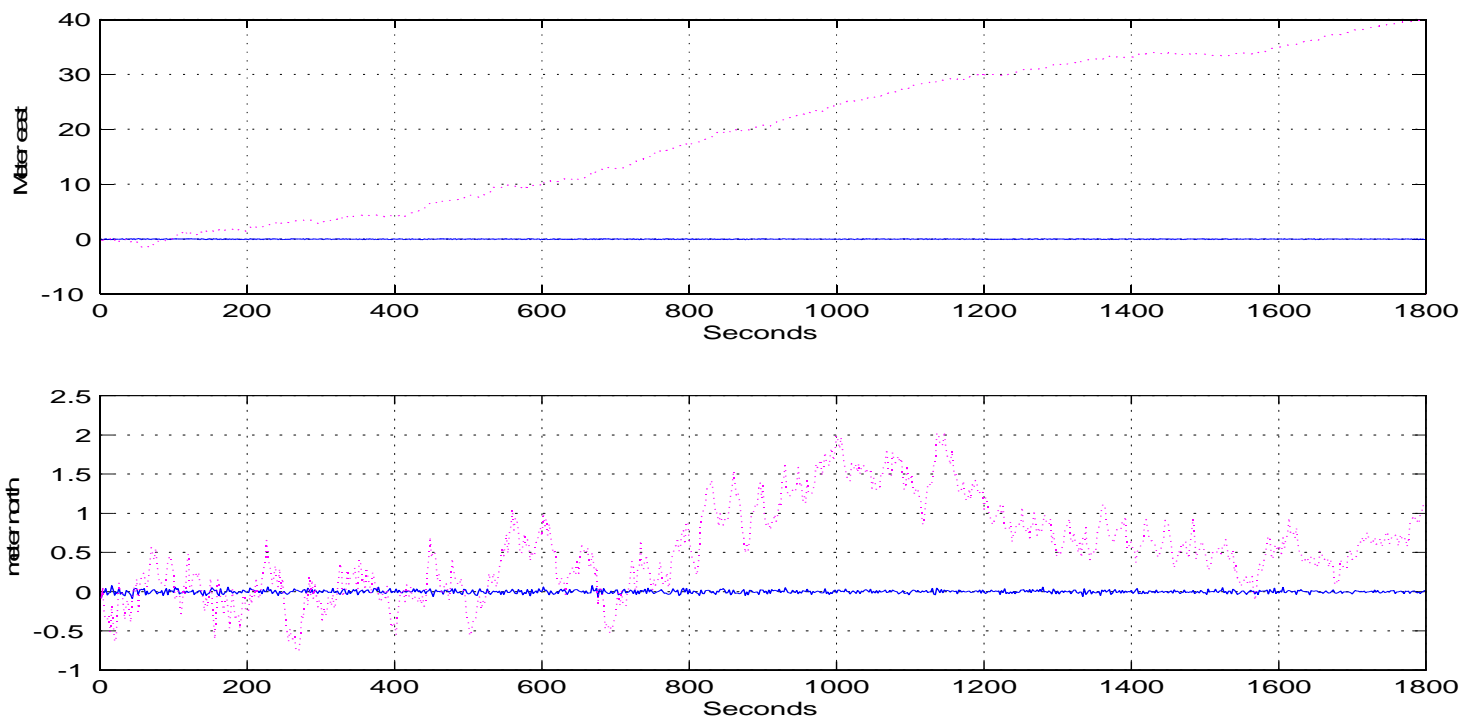

Figure 10 Total system position error of simulation 1. Dotted: true positioning error. Solid: the Kalman filter estimate of the position error.

In Figure 10 the Kalman filter's estimate of the position error is close to zero, making the estimation error the only visible graph. This is due to the reset of INS at each timestep (which in this simulation equals 25 times per second). The mathematical function depicted in Figure 4 shows that the error of the predicted position of an image token is a function of the INS error developed in the time period between the two images. By resetting the INS for each new image, we can guarantee the total INS error to be optimally close to the INS error developed in the time period between two images.

Simulation 2, where the vehicle height is 3000 meters above mean sea level, is shown in figure 11 .
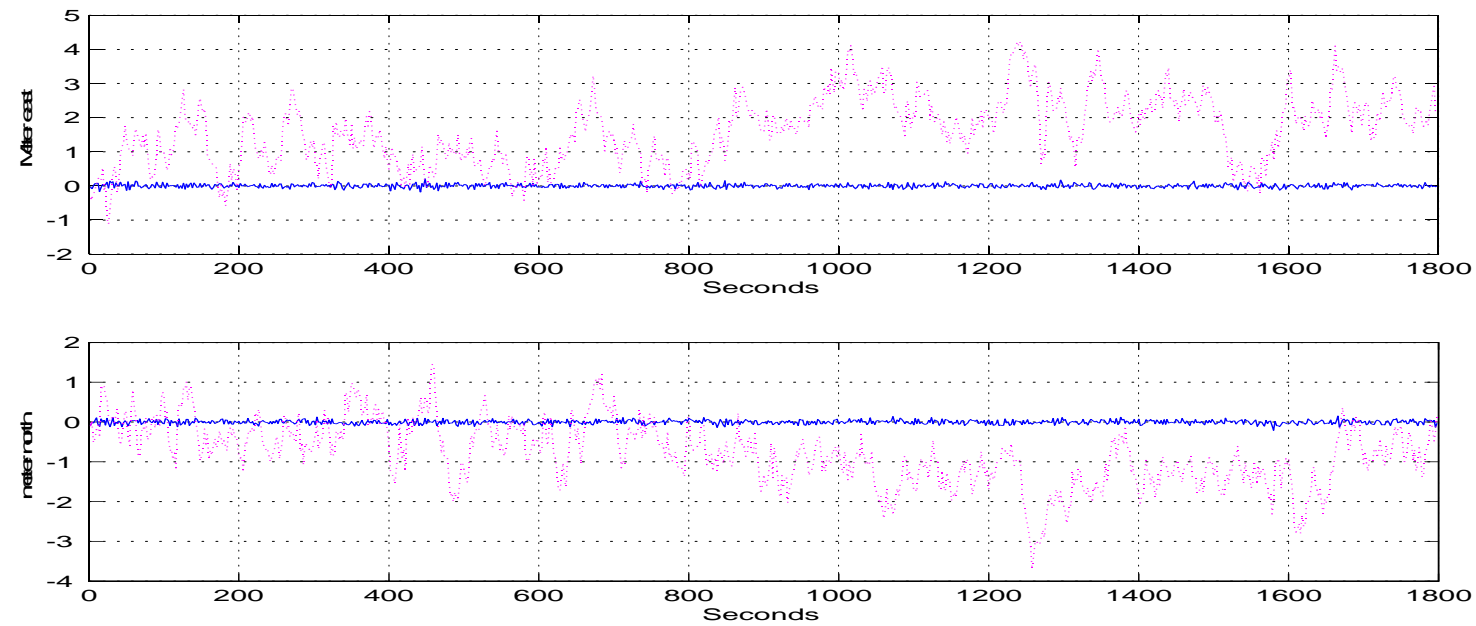

Figure 11 Total system position error of simulation 2. Dotted: true positioning error. Solid: the Kalman filter estimate of the position error. 
Figure 11 shows improved position accuracy compared to Figure 10. Increasing the distance between the vehicle and the terrain increases the time that each landmark is present in the picture. With a constant picture frequency, this gives more measurements of each landmark before we loose it.

Both simulations show more drift in the cross-track direction (east) than in the along-track direction (north). The heading uncertainty is normally the main drift source, giving drift of first order across the trajectory, and of second order along the trajectory.

\subsection{System behaviour}

Increasing the picture frequency provides more measurements of each landmark before its corresponding image token is lost (i.e. before it is out of sight of the camera). With all other parameters unchanged, this reduces the influence of the measurement noise, thereby increasing the navigation accuracy.

Increasing the number of pixels in the image increases the picture resolution, thereby decreasing the measurement noise.

Enlarging the camera aperture angle increases the time period that each image token is present in the picture, thereby allowing more measurements of each landmark, which would improve the total system accuracy. Increasing the camera aperture angle however, results in a wider angle covered by each pixel, so that the measurement noise (in pixels) corresponds to a larger area on the ground, which decreases the total system accuracy.

Changing the camera orientation from pointing straight downward to $45^{\circ}$ forward reduces the system drift. This increases the time that each landmark is in the line-of-sight of the camera, contributing to an increased number of measurements of each landmark.

A curvy terrain beneath the vehicle improves the geometrical distribution of the landmarks, making it easier to solve ambiguities such as roll vs. position in the cross-track direction and pitch vs. position in the along-track direction. Simulations in Hafskjold [5] show that this improves the camera-based navigation performance.

\section{Conclusions}

A method for aiding an INS with a camera-based navigation system has been proposed.

This paper has discussed the effect of system parameters on the navigation performance. Hafskjold [5] showed that the total system accuracy can be improved by:

- Increased picture frequency

- Increased number of landmarks (this requires more computing power, since each new landmark adds 3 elements to the Kalman filter state vector).

- Decreased picture measurement noise (e.g. by avoiding areas covered in mist or smoke, decreasing the camera aperture angle or increasing the picture resolution by increasing the number of pixels in the image). 
- Increased time period in which each image token is present in the image (e.g. by reducing the vehicle speed, increasing the vehicle height above the terrain or increasing the camera aperture angle).

- Increased geometrical distribution of the landmarks (e.g. by choosing a curvy terrain or by reducing the vehicle height above the terrain).

The INS system in the simulations has been of the $>10 \mathrm{nmi} / \mathrm{h}$ class, which is usually found in missiles, UAVs etc. Military and civilian aeroplanes are usually equipped with the more accurate $1 \mathrm{nmi} / \mathrm{h}$ type of systems. A $1 \mathrm{nmi} / \mathrm{h}$ based system will have higher accuracy or alternatively put less demand on the camera-based navigation system aiding.

\section{References:}

[1] P. E. Hagen, "Navigation by images", Modelling, Identification and Control, vol. 14, no.3, 1993.

[2] K. Gade, Integrering av treghetsnavigasjon i en autonom undervannsfarkost (in Norwegian), Institutt for teknisk kybernetikk, Norwegian University of Science and Technology, Norway, 1997.

[3] K. R. Britting, Inertial navigation systems analysis, Wiley Interscience, 1971

[4] D. H. Titterton and J. L. Weston, Strapdown inertial navigation technology, Peter Peregrinus Ltd, Herts, U.K., 1997.

[5] B. H. Hafskjold, Integrert bildebasert navigasjon (in Norwegian), FFI/Rapport-99/02703, Norwegian Defence Research Establishment, Norway, 1999. 